\title{
Assessing cellulose micro/nanofibre morphology using a high throughput fibre analysis device to predict nanopaper performance
}

\author{
Jordan Pennells $\mathbb{D} \cdot$ Bérénice Heuberger • Céline Chaléat $\mathbb{~}$ - Darren J. Martin $\mathbb{D}$
}

Received: 9 November 2021 / Accepted: 23 December 2021 / Published online: 9 February 2022

(C) The Author(s) 2022

\begin{abstract}
Characterising cellulose nanofibre (CNF) morphology has been identified as a grand challenge for the nanocellulose research field. Direct techniques for CNF morphology characterisation exhibit various difficulties related to the material network structure and equipment cost, while indirect techniques that investigate fibre-light interaction, fibre-solvent interaction, fibre-fibre interaction, or specific fibre surface area involve relatively facile methods but may be more unreliable. Nanopaper mechanical testing is a prevalent metric for assessing fibre-fibre interaction, but is an off-line, time-consuming, and destructive methodology. In this study, an optical fibre morphology analyser (MorFi, Techpap) was employed as an on-line, high throughput, fast turnaround tool to assess micro/nanofibre pulp morphology and predict the properties of nanopaper material. Correlation analysis identified fibre content and fibre kink properties as most correlated with nanopaper strength and
\end{abstract}

Supplementary Information The online version contains supplementary material available at https://doi.org/10.1007/ s10570-021-04405-5.

J. Pennells $(\bowtie) \cdot$ C. Chaléat · D. J. Martin

School of Chemical Engineering, The University of

Queensland, Brisbane, QLD 4072, Australia

e-mail: jordan.pennells@uq.net.au

B. Heuberger

Axiom Project Services Pty Ltd, 300 Queen St, Brisbane,

QLD 4000, Australia toughness, while fibre width and coarseness were most inversely correlated with nanopaper performance. Principal component analysis (PCA) was employed to visualise interdependent morphological and mechanical data. Subsequently, two data driven statistical models-multiple linear regression (MLR) and machine learning based support vector regression (SVR) — were established to predict nanopaper properties from fibre morphology data, with SVR generating a more accurate prediction across all nanopaper properties $(\mathrm{NRMSE}=0.13-0.33)$ compared to the MLR model (NRMSE $=0.33-0.51)$. This study highlights that statistical methods are useful to disentangle and visualise interdependent morphological data from an on-line fibre analysis device, while regression models are also capable of predicting paper mechanical properties from CNF samples even though these devices do not operate at nanoscale resolution. 


\section{Graphical abstract}

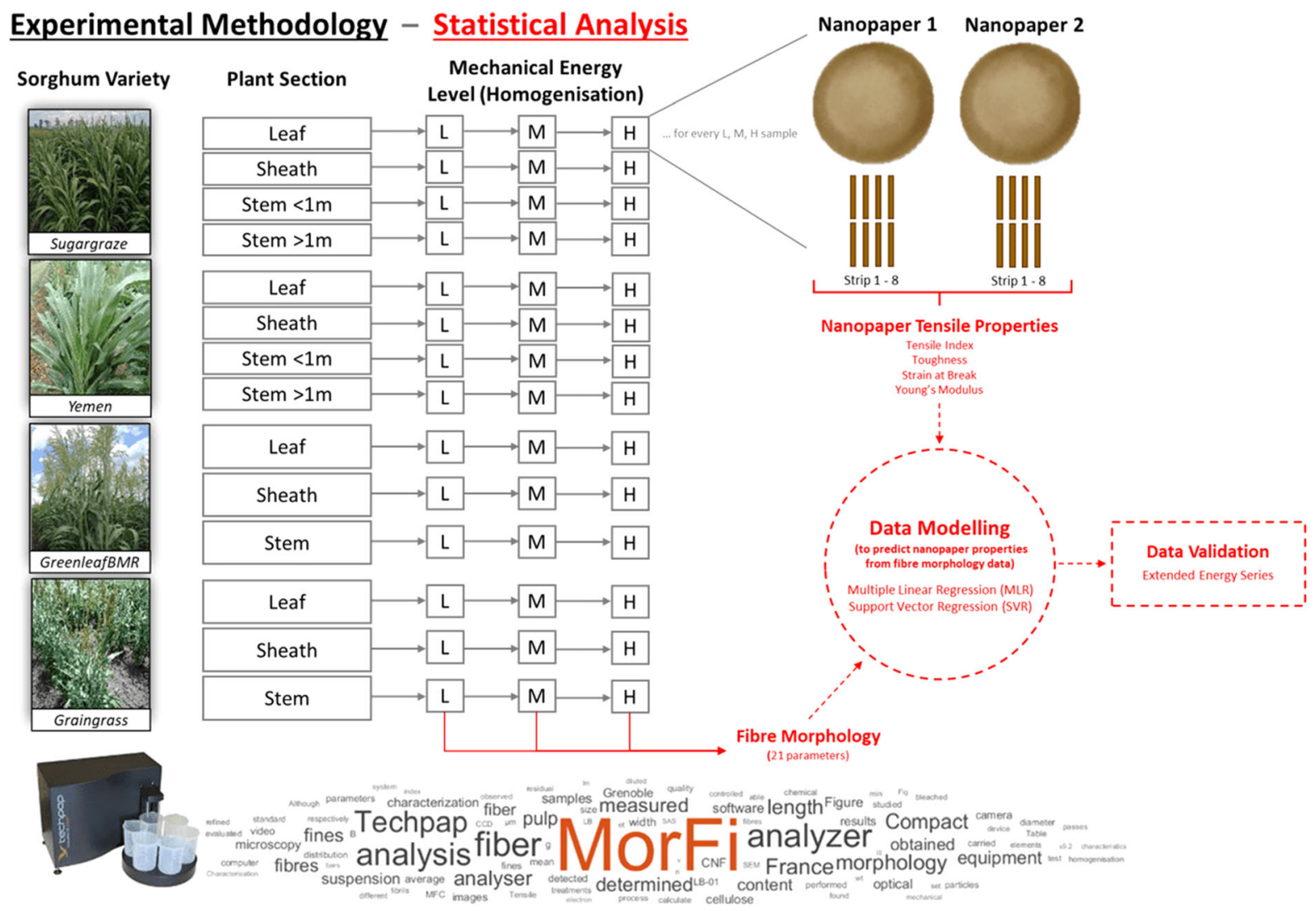

Keywords Cellulose nanofibers - Fibre morphology · Nanopaper · Support vector regression . Principal component analysis $\cdot$ Sorghum

\section{Introduction}

Characterising CNF morphology has been identified as a grand challenge for nanocellulose research (Moon et al. 2011). Across various nanocellulose applications, it is an important parameter for assessment of product quality, quality control, and material safety (Campano et al. 2020). Fibre morphology encompasses the average fibre dimensions (length, width), the relative size distribution of fibre dimensions throughout the sample, fibre aspect ratio, fibre surface area, the degree of fibrillation and branching, fibre hydrodynamic volume (rigidity), and fibre shape (kink, curl, curvature). Subsequently, nanofibre morphology influences the effective nanofibre surface area, fibre-fibre and fibre-solvent interaction, net surface charge, gel point or networking concentration, and therefore the product quality in terms of its mechanical properties, rheological and colloidal behaviour, hydrophilicity, optical properties, electrical conductivity, film permeability, and material reactivity ( $\mathrm{Li}$ et al. 2021). Fibre morphology has a well-established impact on the mechanical properties of paper, such that fibre length strongly affects paper strength, whereas fibre width decreases fibre flexibility and conformability, which has a negative impact on paper strength (Seth 1995; Larsson et al. 2018). Fine content improves the strength, smoothness, and optical properties of the final paper (Moral et al. 2010; Motamedian et al. 2019), while decreasing the freeness of the pulp (Dienes et al. 2005). 
Analysing cellulose nanofibre morphology

Measuring fibre morphology has been a difficult task in nanocellulose research and development, primarily due to nanocellulose materials existing over a range of length scales, from poorly fibrillated millimetric scale cellulose bundles to micron scale cellulose microfibres (CMF), and down to nanoscale fibrillated cellulose nanofibres (Tanaka et al. 2012; Chinga-Carrasco 2013). This length scale range introduces a challenge for capturing a representative, bulk analysis of fibre morphology. Other challenges for characterising CNF morphology include the interconnected network structure of the material, and the difficulty of observing nanofibres in their native, aqueous suspended state (Haapala et al. 2013).

Many tools have been proposed to address the challenge of CNF morphology characterisation, including both direct observational measurement of fibres such as SEM, TEM, and AFM (Krishnamachari et al. 2011; Campano et al. 2020), or indirect measurement of related metrics that are known to be influenced by nanofibre morphology, such as light transmittance and scattering (Chinga-Carrasco 2013; Moser et al. 2015; Shimizu et al. 2016), freeness or drainage time (Dienes et al. 2005), fractionation (Madani et al. 2011; Tanaka et al. 2012), crystallinity (Qing et al. 2013), gel point (Varanasi et al. 2013; Sanchez-Salvador et al. 2020), rheology (Li et al. 2015; Yuan et al. 2021), and specific fibre surface area (Pääkko et al. 2007; Saito et al. 2007).

Both direct and indirect fibre morphology characterisation exhibit difficulties. Direct nanofibre characterisation enables high resolution visualization of individual particles or aggregates at multiple magnification levels, but is limited in its ability to analyse a representative sample of the material in a timely manner and for fibres across multiple length scales (Legland and Beaugrand 2013). While the resolution of TEM can analyse fibre width down to a few nanometres, SEM is only capable of analysing fibre width greater than $100 \mathrm{~nm}$ (Kangas et al. 2014). In addition, the three-dimensional structure and bulk morphology of the sample is disrupted during the drying step required for sample preparation (Peng et al. 2012; Silva et al. 2021). Meanwhile, indirect characterisation involves measuring a derived property of the nanofibre system, such as the fibre-light interaction (DLS, UV-vis transmittance), fibre- solvent interaction (rheology, sedimentation behaviour, water retention capacity), fibre-fibre interaction (nanopaper mechanical properties), or specific surface area (SANS, SAXS, DSC, BET adsorption, solvent relaxation NMR, conductimetric titration). Typically, calculations or models are employed to infer fibre morphology characteristics. These tools generally involve more simple methods of nanofibre characterisation but are often unreliable due to potential inaccuracy and lack of generalisation in the models inferring nanofibre properties. This issue is emphasised in the nanocellulose field, where different biomass sources and processing methodologies can produce material ranging widely in terms of fibre morphology and mechanical properties.

As nanocellulose materials become increasingly commercial, there is strong incentive to shift from laboratory scale characterisation methods of fibre morphology to scalable, fully automated, on-line characterisation systems that are capable of assessing thousands of fibre elements over a relatively short time period (Legland and Beaugrand 2013; Balea et al. 2021).

Fibre analysis tools in nanocellulose literature

Optical fibre analysis devices are a potential solution for this characterisation challenge. These tools have the potential to provide high throughput, fast turnaround analysis of micro and nanofibre pulp morphology. Commercial fibre analysis devices have previously been compared with varying results depending on their fibre analysis algorithm (Guay et al. 2005; Turunen et al. 2005; Hirn and Bauer 2006). One such commercial fibre analysis device is the MorFi (Techpap, France), which has been selected as the fibre analysis system for this study. Although MorFi is designed to analyse fibres in the size range produced within the pulp and paper industry, many studies have been conducted with MorFi as an indirect nanofibre characterisation tool, as shown in Table 1.

Typically, MorFi is used to add another facet of characterisation for CNF pulp, but the fact that it cannot detect nano-scale fibre dimensions is rarely taken into account (Espinosa et al. 2020). Alternatively, MorFi has also been used as a quality control tool to inform when sufficient mechanical processing has been applied to the sample to reach a desired morphology setpoint. Less commonly, MorFi been 
used to conduct detailed characterisation of the nanofibre pulp morphology (Lacerda et al. 2013; Rol et al. 2018), and integrating results with additional characterisation methods to assess emergent relationships (Rol et al. 2019; Espinosa et al. 2020). This style of investigation is valuable, because information obtained at the fibre population level can greatly aid process development strategies and product quality monitoring (Haapala et al. 2013).

\section{Predicting nanopaper quality}

Nanopaper fabrication and testing is one of the predominant methods to characterise cellulose nanofibre quality. It is well established that nanopaper mechanical performance is correlated to the degree of fibrillation and nano-scale morphology of CNF pulp. Although nanopaper fabrication and testing is a relatively facile method for $\mathrm{CNF}$ characterisation (TAPPI 2006), it is necessarily off-line, time-consuming, and destructive (Aguado et al. 2016). In addition, nanopaper mechanical characterisation can suffer from low precision due to defects introduced during the sample cutting procedure (Hervy et al. 2017). As such, a model that is capable of predicting nanopaper performance based off of predictor variables, such as fibre morphology data, is advantageous for CNF research and development (García-Gonzalo et al. 2016). In addition, conventional CNF characterisation typically involves off-line measurement techniques performed in a laboratory setting, which generally require high capital investment, highly qualified personnel for device operation, and involve complex and time-consuming post-processing and analysis of data (Balea et al. 2021). Alternatively, a fibre morphology analysis device could be employed as an on-line quality control tool for continuous CNF processing, with its simple operation, fast analysis, and low cost encouraging the commercialisation of CNF products (Aguado et al. 2016). While the integration of fibre analysis tools with pulp and paper production would greatly benefit CNF commercialisation, it has only been addressed in a handful of previous studies (Pande and Roy 1998; Oluwafemi and Sotannde 2007; Lin et al. 2014; Nasser et al. 2015; Aguado et al. 2016). To address this gap in the literature, this study investigates the relationships between fibre morphology and nanopaper properties for a broad sample population consisting of different varieties and sections of sorghum biomass-a globally important agricultural crop (Borrell et al. 2021) processed under 3 different energy levels. A statistical methodology has been undertaken, including correlation analysis, principal component analysis, and regression modelling, to assess the relationships between fibre morphology parameters and nanopaper mechanical properties, and to predict nanopaper performance from fibre morphology data.

\section{Experimental methodology}

\section{Materials}

Sodium hydroxide $(\mathrm{NaOH}$, pellets, $98 \%$ purity) was purchased from Chem Supply, Australia. Reverse

Table 1 Literature publications involving MorFi for CNF characterisation

\begin{tabular}{|c|c|c|}
\hline Publication type & $\begin{array}{l}\text { Number of } \\
\text { publications }\end{array}$ & Description \\
\hline Characterisation & 104 & General characterisation of nanofibre pulp morphology \\
\hline Setpoint measurement & 11 & Fibre morphology used to determine when a refining setpoint has been reached \\
\hline Commentary & 4 & Commentary about the use of MorFi as a fibre analysis tool \\
\hline Review & 3 & Comparative review of different fibre analysis tools \\
\hline $\begin{array}{l}\text { Detailed } \\
\text { characterisation }\end{array}$ & 2 & Detailed characterisation of nanofibre pulp morphology \\
\hline $\begin{array}{l}\text { Integrated } \\
\text { characterisation }\end{array}$ & 2 & $\begin{array}{l}\text { Characterisation of nanofibre pulp morphology, Integrated with other nanofibre } \\
\text { characterisation tools }\end{array}$ \\
\hline Methodology & 1 & Discussion around the methodology for conducting MorFi analysis \\
\hline
\end{tabular}


osmosis (RO) purified water was used throughout experimentation. Sorghum biomass was grown under adequate nutrient and water input conditions at The University of Queensland's research farm at the Gatton campus near Brisbane, QLD from seeds provided by the QLD Department of Agriculture and Fisheries (DAF). Sorghum biomass included four varieties (Sugargraze, Yemen, GreenleafBMR, Graingrass) partitioned into four plant sections (Leaf, Sheath, Stem $<1 \mathrm{~m}$, Stem $>1 \mathrm{~m}$ ).

\section{Biomass preparation \& CNF production}

Biomass was cut into approximately $5 \mathrm{~cm}$ lengths, washed three times in distilled water at $80{ }^{\circ} \mathrm{C}$ for approximately $30 \mathrm{~min}$, and subsequently dried in a convection oven at $\sim 55{ }^{\circ} \mathrm{C}$ for 3 days. Dried biomass was ground using a Retsch SM300 mill (Retsch $\mathrm{GmbH}$, Germany) at $3000 \mathrm{rpm}$ with a $1 \mathrm{~mm}$ trapezoidal mesh screen. Oversized material was separated from the ground biomass with a $0.71 \mathrm{~mm}$ aperture sieve. Ground biomass was dispersed and stirred overnight at $\sim 350 \mathrm{rpm}$ in deionised water at a solid ratio of 1:20 (20 $\mathrm{g}$ of water for every $1 \mathrm{~g}$ of ground biomass). Chemical pretreatment was performed using a $2 \% \mathrm{NaOH}$ solution $(\mathrm{w} / \mathrm{v})$ at $80{ }^{\circ} \mathrm{C}$ for $2 \mathrm{~h}$, stirred at $\sim 350 \mathrm{rpm}$. $\mathrm{NaOH}$ treated (delignified) pulp was separated from the waste liquor through a fine mesh sieve (53 $\mu \mathrm{m}$ aperture) and rinsed extensively until the filtrate $\mathrm{pH}$ was below 8 . The delignified pulp suspension was diluted to a setpoint of $0.5 \%$ (w/ v) using a Mettler Toledo moisture analyser.

High pressure homogenisation

High pressure homogenisation (HPH) was conducted with a Niro-Soavi Panda Plus 2000 machine (GEA, Italy). Samples were collected at three sequential energy levels: Low energy (L) $-1 \times 400$ bar pass, Medium energy (M) $-1 \times 400$ bar pass $+1 \times 700$ bar pass, High energy $(\mathrm{H})-1 \times 400$ bar pass $+1 \times$ 700 bar pass $+2 \times 1100$ bar passes.

Fibre morphology analysis

$10 \mathrm{~g}$ of CNF suspension at $0.5 \%(\mathrm{w} / \mathrm{v})$ was randomly sampled and added to approximately $1 \mathrm{~L}$ of water for fibre analysis using the MorFi Compact analyser (Techpap, France) equipped with CCD video camera, a high magnification optical flow cell and MorFi R.10.07 automatic analysis software. According to the default settings of the MorFi device, Fibres are classified as elements with a length between 100 and $10 \mathrm{~mm}$ and a width between 5 and $75 \mu \mathrm{m}$, while Fine elements have a length between 5 and $100 \mu \mathrm{m}$ and a width $<5 \mu \mathrm{m}$. Material below the optical resolution of this device (length $<5 \mu \mathrm{m}$ ) were not captured in the analysis. Four technical replicates were run for each sample. The MorFi parameters are outlined in Table 2.

Nanopaper mechanical properties

Nanopaper was prepared from CNF pulp according to our previous work (Pennells et al. 2021), as described in brief below. CNF pulp was dewatered and dried into a nanopaper handsheet using an automatic Rapid Köthen handsheet former (Xell, Austria) according to the ISO 5269-2:2004 standard operating procedure (International Organization for Standardization 2001). First, the CNF suspension was dewatered under $0.7 \mathrm{kPa}$ vacuum until sufficiently dry, as assessed with a P-2000 handheld moisture analyser (Delmhorst, USA). Subsequently, the wet cake was dried under vacuum at $110{ }^{\circ} \mathrm{C}$ for $20 \mathrm{~min}$. Nanopaper handsheets were conditioned in the laboratory $\left(25^{\circ} \mathrm{C}, 50-75 \%\right.$ $\mathrm{RH})$ for at least 1 day prior to mechanical testing. Up to eight rectangular nanopaper strips $(\mathrm{L}=150 \mathrm{~mm}$, $\mathrm{W}=15 \mathrm{~mm}$ ) were cut out from each handsheet, and up to two handsheets were prepared for each sample. Tensile properties of each nanopaper strip were measured using an Instron model 5543 universal testing machine (Instron Pty Ltd., Melbourne, Australia) equipped with a $500 \mathrm{~N}$ load cell. Tensile index was calculated with Eq. 1, where $\sigma_{\mathrm{w}}^{\mathrm{T}}$ is the tensile index or specific tensile strength per unit weight in $\mathrm{Nm} \cdot \mathrm{g}^{-1}$, UTS is the ultimate tensile strength in $\mathrm{Pa}$, and $\rho_{n}$ is the nanopaper density in $\mathrm{kg} \cdot \mathrm{m}^{-3}$.

$\sigma_{\mathrm{w}}^{\mathrm{T}}=\frac{\operatorname{UTS}(\mathrm{Pa})}{\rho_{\mathrm{n}}}$

Toughness was calculated as a numerical approximation of the energy absorbed by the nanopaper strip, according to Eq. 2, where $\mathrm{U}_{\mathrm{T}}$ is the toughness in $\mathrm{MJ} \cdot \mathrm{m}^{-3}, 0$ is the zero-strain starting point, and $\epsilon_{\mathrm{f}}$ is the nanopaper failure strain. 
Table 2 MorFi output parameters and parameter descriptions

\begin{tabular}{|c|c|c|c|}
\hline Code & Parameter & Unit & Description \\
\hline Fibre_n & $\begin{array}{l}\text { Number of analysed } \\
\text { fibres }\end{array}$ & - & The total number of fibres analysed throughout the test \\
\hline Fibre_cont & Fibre content & Millions/g of pulp & The number of fibres (millions) per gram of sample analysed \\
\hline Fibre_L & Mean arithmetic length & $\mu \mathrm{m}$ & Arithmetic mean length of all fibres analysed \\
\hline Fibre_L.L & $\begin{array}{l}\text { Mean length-weighted } \\
\text { length }\end{array}$ & $\mu \mathrm{m}$ & $\begin{array}{l}\text { Arithmetic mean length of all fibres analysed, weighted by fibre } \\
\text { length }\end{array}$ \\
\hline Fibre_L3.L & $\begin{array}{l}\text { Mean cubic-length- } \\
\text { weighted length }\end{array}$ & $\mu \mathrm{m}$ & $\begin{array}{l}\text { Arithmetic mean length of all fibres analysed, weighted by the } \\
\text { cube of the fibre length }\end{array}$ \\
\hline Fibre_A.L & $\begin{array}{l}\text { Mean area-weighted } \\
\text { length }\end{array}$ & $\mu \mathrm{m}$ & $\begin{array}{l}\text { Arithmetic mean length of all fibres analysed, weighted by fibre } \\
\text { area }\end{array}$ \\
\hline Fibre_W & Mean fibre width & $\mu \mathrm{m}$ & Arithmetic mean width of all fibres analysed \\
\hline Fibre_coarse & $\begin{array}{l}\text { Mean fibre coarseness. } \\
\mathrm{mg} / \mathrm{m}\end{array}$ & $\mathrm{mg} / \mathrm{m}$ & Fiber mass per unit length \\
\hline Fibre_kink.num & Mean kink number & - & $\begin{array}{l}\text { The number of fibres with small regions of very high curvature } \\
\text { (i.e. sharp bends) along the fiber }\end{array}$ \\
\hline Fibre_kink.ang & Mean kink angle & & The mean kink angle for fibres that are kinked \\
\hline Fibre_kink.cont & Kinked fibre content & $\%$ & The number percentage of fibres with a kinked structure \\
\hline Fibre_curl & Mean fibre curl index & $\%$ & $\begin{array}{l}\text { Degree of nonstraightness of a fiber-or the ratio of total particle } \\
\text { length over the projected length }\end{array}$ \\
\hline MF.index & $\begin{array}{l}\text { MacroFibrillation } \\
\text { index }\end{array}$ & $\%$ & Percentage of microfibrils by area \\
\hline Fibre_broken & Broken fibre content & $\%$ & Number percentage of fibres with broken ends \\
\hline Fine_n & $\begin{array}{l}\text { Number of analysed } \\
\text { fines }\end{array}$ & - & The total number of fibres analysed throughout the test \\
\hline Fine_cont & Fine content & Millions/g of pulp & The number of fines (millions) per gram of sample analysed \\
\hline Fine_cont.A & Fine content & $\%$ (in area) & $\%$ Area of Fine Elements over all other elements \\
\hline Fine_cont.L & Fine content & $\%$ (in length) & $\%$ Length of Fine Elements over all other elements \\
\hline Fine_cont.L.L & Fine content & $\begin{array}{l}\% \text { (in length- } \\
\text { weighted length) }\end{array}$ & $\begin{array}{l}\text { \% Length weighted length of Fine Elements over all other } \\
\text { elements }\end{array}$ \\
\hline Fine_A & Mean fine area & $\mu \mathrm{m}^{2}$ & Arithmetic mean fine area \\
\hline Fine_L & Mean fine length & $\mu \mathrm{m}$ & Arithmetic mean fine length \\
\hline
\end{tabular}

$U_{T}=\int_{0}^{\epsilon_{f}} f(x) d x \approx \sum_{i=2}^{n} \frac{1}{2}\left[x_{n}-x_{n-1}\right]\left[f\left(x_{n}\right)+f\left(x_{n-1}\right)\right]$

\section{Statistical methodology}

\section{Correlation analysis}

Following the generation and compilation of fibre morphology data, for which the four technical replicates for each sample were calculated into a mean, two
Pearson's correlation matrices were built to assess the relationships between fibre morphology parameters and nanopaper mechanical properties for all sorghum varieties, sections, and energy levels. The two correlation matrices included: (1) the correlation between each fibre morphology parameter, and (2) the correlation between each fibre morphology parameter and each of the four nanopaper mechanical properties.

\section{Principal component analysis}

Principal component analysis (PCA) was performed using the prcomp command of the $\mathrm{R}$ statistical 
software (R Core Team 2021) with RStudio v1.3.1056 in order to reduce the dimensionality of the fibre morphology and nanopaper mechanical property data sets. Normal data probability ellipses were fitted for the different factors (variety, section, energy level) based on the default normal probability of $68 \%$ (Prager et al. 2020). The top two principal components were selected for data visualisation.

\section{Regression modelling}

To predict nanopaper properties, the fibre morphology parameters outputs were related to the nanopaper properties using one of two data modelling options: Multiple linear regression (MLR) or machine learning based support vector regression (SVR) in MatLab v.2021a using the libraries developed by Pedregosa et al. (Pedregosa et al. 2011). The SVR hyperparameters selected included the kernel function of 2 nd degree polynomial, the absolute value of the $\alpha$ coefficient equal to the IQR/1.349, and $\varepsilon$ equal to $\mathrm{IQR} / 13.49$, where IQR is the interquartile range. Each of the SVR models was fivefold cross validated, with the final correlation coefficient $\mathrm{R}^{2}$ corresponding to the average of the five values calculated for each fold. The accuracy of each model was assessed for each nanopaper metric based on their $\mathrm{R}^{2}$ and root mean squared error (RMSE) values. RMSE is the square root of the mean of squared errors between the observed and predicted values, as shown in Eq. 3

$R M S E=\sqrt{\frac{\sum_{i-1}^{n}\left(O_{i}-P_{i}\right)}{n}}$

where $\boldsymbol{O}_{\boldsymbol{i}}$ and $\boldsymbol{P}_{\boldsymbol{i}}$ represent the observed and predicted values for each sample (of size $\boldsymbol{n}$ ) (Ritter and MuñozCarpena 2013). RMSE was normalised by the mean value of each nanopaper metric.

Model validation

For model validation, an additional series of CNF suspension was prepared through HPH processing over an extended mechanical energy series. The biomass sample used for this analysis was the Sugargraze variety with all sections combined in equal proportions. CNF pulp was prepared according to the mechanical processing conditions outlined in Table 3. Nanopaper handsheets were fabricated from the prepared CNF pulp and tested in accordance with the previously described experimental methodology. A graphical overview of the experimental methodology and statistical analysis employed in this study is presented in the Graphical Abstract.
Table 3 Model validation data set over an extended $\mathrm{HPH}$ processing series

\begin{tabular}{lll}
\hline Energy Level & Notation & HPH Passes \\
\hline Low-Low & LL & $200 \mathrm{bar}$ \\
Low & $\mathrm{L}$ & $200 \mathrm{bar}+400 \mathrm{bar}$ \\
Low-Medium & $\mathrm{LM}$ & $200 \mathrm{bar}+400 \mathrm{bar}+550 \mathrm{bar}$ \\
Medium & $\mathrm{M}$ & $200 \mathrm{bar}+400 \mathrm{bar}+550 \mathrm{bar}+700 \mathrm{bar}$ \\
Medium-High & $\mathrm{MH}$ & $200 \mathrm{bar}+400 \mathrm{bar}+550 \mathrm{bar}+700 \mathrm{bar}+1 \times 1100 \mathrm{bar}$ \\
High & $\mathrm{H}$ & $200 \mathrm{bar}+400 \mathrm{bar}+550 \mathrm{bar}+700 \mathrm{bar}+2 \times 1100 \mathrm{bar}$ \\
High-High & $\mathrm{HH}$ & $200 \mathrm{bar}+400 \mathrm{bar}+550 \mathrm{bar}+700 \mathrm{bar}+3 \times 1100 \mathrm{bar}$ \\
\hline
\end{tabular}




\section{Results}

Fibre Morphology Correlations

Initially, a correlation matrix detailing the relationship between each pair of fibre morphology parameters was calculated, as displayed in Fig. 1. Using a significance threshold of $\boldsymbol{x} \geq 0.7$, a total of 27 of the 210 pairwise correlations were considered significantly correlated. Relationships of interest included the positive correlation between fibre width (fibre_W), fibre coarseness (fibre_coarse), fibre curl index (fibre_curl), macrofibrillation index (MF.index), and broken fibre content (fibre_broken), the inverse correlation between these parameters and fibre content (fibre_cont), and the inverse correlation between kinked fibre content (fibre_kink.cont) and mean fine area (fine_A) and length (fine_L). Following the analysis of pairwise correlations in Fig. 1, a correlation table was established between each fibre morphology parameter and each of the four nanopaper mechanical performance metrics, as outlined in Table 4. Notable positive correlations included fibre content (fibre_cont) for tensile index and Young's modulus (0.6 and 0.71, respectively), the fine content (fine_cont) for Young's modulus (0.67), and all kink-related parameters for

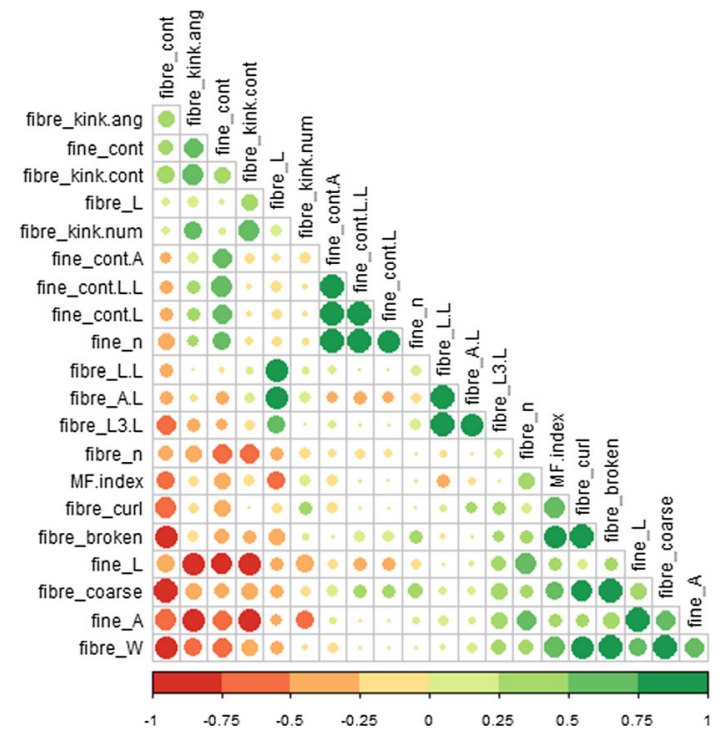

Fig. 1 Fibre morphology parameter correlation matrix

toughness (0.6-0.66). Taking the average correlation across all four nanopaper metrics, kinked fibre content (fibre_kink.cont) and kink angle (fibre_kink.ang) have the highest values ( 0.55 and 0.56 , respectively), indicating the strongest overall relationship to nanopaper performance out of all fibre morphology parameters. The most indicative fibre morphology parameter

Table 4 Pearson correlation values for each fibre morphology parameter across the four nanopaper metrics

\begin{tabular}{|c|c|c|c|c|}
\hline Fibre Morphology Parameter & TI & Toughness & $\begin{array}{c}\text { Strain at } \\
\text { break }\end{array}$ & $\begin{array}{l}\text { Young's } \\
\text { Modulus }\end{array}$ \\
\hline Fibre content (millions/g of pulp) & 0.60 & 0.44 & 0.20 & 0.71 \\
\hline Average kink angle $\left(^{\circ}\right)$ & 0.47 & 0.60 & 0.45 & 0.71 \\
\hline Kinked fibre content (\%) & 0.46 & 0.66 & 0.54 & 0.56 \\
\hline Average kink number & 0.43 & 0.63 & 0.59 & 0.37 \\
\hline Mean arithmetic length $(\mu \mathrm{m})$ & 0.27 & 0.30 & 0.35 & 0.11 \\
\hline Fine content (millions/g of pulp) & 0.19 & 0.26 & 0.08 & 0.67 \\
\hline Mean area-weighted length $(\mu \mathrm{m})$ & 0.07 & 0.16 & 0.32 & -0.28 \\
\hline Mean length-weighted length $(\mu \mathrm{m})$ & 0.03 & 0.11 & 0.24 & -0.17 \\
\hline Number of analysed fibres & -0.03 & -0.12 & -0.01 & -0.39 \\
\hline Fine content (\% length) & -0.11 & 0.03 & -0.01 & 0.28 \\
\hline Fine content ( $\%$ area $)$ & -0.12 & -0.03 & -0.07 & 0.27 \\
\hline Fine content (\% length weight. length) & -0.13 & 0.04 & -0.01 & 0.28 \\
\hline Number of analysed fines & -0.14 & 0.02 & 0.02 & 0.16 \\
\hline Mean cubic-length.-weigh. length $(\mu \mathrm{m})$ & -0.19 & -0.10 & 0.08 & -0.42 \\
\hline MacroFibrillation index (\%) & -0.19 & -0.13 & -0.01 & -0.37 \\
\hline Mean fine length $(\mu \mathrm{m})$ & -0.36 & -0.50 & -0.33 & -0.69 \\
\hline Mean fibre curl index (\%) & -0.44 & -0.18 & -0.02 & -0.56 \\
\hline Mean fine area $\left(\mu \mathrm{m}^{2}\right)$ & -0.47 & -0.53 & -0.34 & -0.71 \\
\hline Broken fibre content (\%) & -0.54 & -0.34 & -0.17 & -0.58 \\
\hline Mean fibre width $(\mu \mathrm{m})$ & -0.66 & -0.51 & -0.30 & -0.80 \\
\hline Mean fibre coarseness (mg/m) & -0.71 & -0.52 & -0.32 & -0.73 \\
\hline
\end{tabular}


HPH Energy Level $\rightarrow \mathrm{L} \rightarrow \mathrm{M} \rightarrow \mathrm{H}$
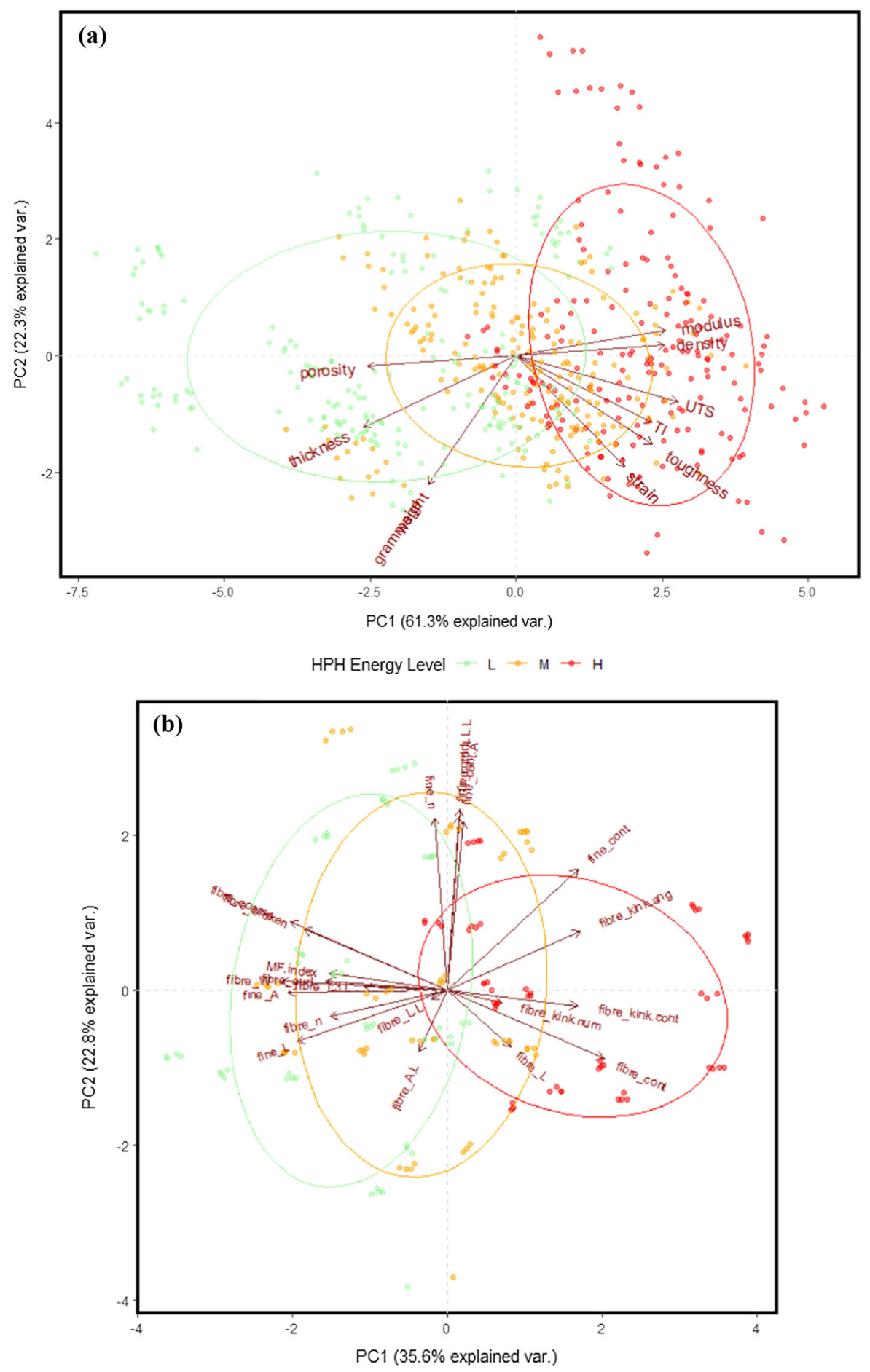

Fig. 2 Principal Component Analyses for: a the association between Nanopaper properties grouped by HPH energy level; and b the association between fibre morphology parameters grouped by HPH energy level 
for a single nanopaper property was the inverse correlation between Young's modulus and mean fibre width $(-0.8)$. Other inverse correlations between fibre morphology parameters and nanopaper properties include fibre coarseness (fibre_coarse) for all nanopaper metrics besides strain at break ( -0.51 to -0.8$)$ and mean fine length (fine_L) and mean fine area (fine_A) for Young's modulus $(-0.69$ and -0.71 , respectively).

\section{Principal component analysis (PCA)}

Following the correlation analysis, a series of PCAs were run to visualise the grouping and variance of fibre morphology data across the entire sample population in a reduced dimensionality format. Initially, a PCA was conducted on the nanopaper mechanical property data across all biomass samples and energy levels (Fig. 2a). The results from the nanopaper PCA demonstrate that the increase in mechanical processing energy from low to high shifted the data along the first principal component, which explains $61.3 \%$ of the overall variance. This shift was closely matched by the shift in nanopaper density and Young's modulus, and to a lesser extent tensile index (TI), toughness and strain at break. This confirms the existing notion that energy level has a strong positive correlation with these nanopaper material properties.

Subsequently, a PCA was conducted on the fibre morphology data across all biomass samples and energy levels (Fig. 2b). The results from the fibre morphology PCA demonstrate the fibre morphology parameters that are correlated and inversely correlated with the mechanical processing energy (along PC1), which are known to relate to the nanopaper properties from Fig. 2a. Fibre kink properties and fibre content are positively associated with processing energy, and therefore nanopaper performance, while parameters such as fibre width, broken fibre content, and fibre coarseness are inversely associated with processing energy and nanopaper performance. Fibre morphology parameters that correlate to PC2 include the fine number and the fine content weighted by area, length, and length-weighted length, respectively. The variance in these parameters is more closely associated with different plant sections, specifically the leaf section, as demonstrated in the Supplementary Material. The two first principal components for the fibre morphology PCA explain $35.6 \%$ and $22.8 \%$ of the total variance, respectively.

\section{Nanopaper regression models}

Prediction of nanopaper mechanical properties from fibre morphology data was performed using two regression modelling techniques: Multiple Linear Regression (MLR) and Support Vector Regression (SVR). For both regression models, fitting of the fibre morphology parameters was assessed for each nanopaper metric based on their $\mathrm{R}^{2}$ and NRMSE values, as seen in Table 5. Based on the MLR and SVR model outputs, it can be concluded that the given MorFi data best explains nanopaper performance in terms of tensile index (NRMSE of 0.4 and 0.23 , respectively) and Young's modulus (NRMSE of 0.33 and 0.13 , respectively). For the given dataset, both the MLR and SVR model outputs demonstrate a high level of accuracy for predicting nanopaper properties from fibre morphology data across all four nanopaper metrics $\left(\mathrm{R}^{2}>0.88\right)$. A full overview of the MLR and SVR regression coefficients and parameters are provided in the Supplementary Material.

However, as the SVR model demonstrated the higher accuracy for predicting nanopaper properties, it was selected for further investigation in the subsequent analyses. Figure 3 portrays the measured and SVR predicted nanopaper tensile index, which demonstrates the high level of accuracy for this model for all sorghum samples across difference varieties, sections, and energy levels.

Considering the impact of the mechanical energy level on the accuracy of the regression model predictions, Table 6 demonstrates that medium and high energy samples had the lowest NRMSE values across the majority of nanopaper metrics (excluding

Table 5 Fibre morphology to nanopaper prediction modelling using MLR and SVR

\begin{tabular}{llllll}
\hline Nanopaper Metrics & \multicolumn{2}{l}{ MLR model } & & \multicolumn{2}{l}{ SVR model } \\
\cline { 2 - 3 } & NRMSE & $\mathrm{R}^{2}$ & & NRMSE & $\mathrm{R}^{2}$ \\
\hline Tensile Index & 0.4 & 0.89 & 0.23 & 0.99 \\
Toughness & 0.4 & 0.88 & 0.28 & 0.98 \\
Strain at break & 0.51 & 0.8 & 0.33 & 0.98 \\
Young's modulus & 0.31 & 0.93 & 0.13 & 0.99 \\
\hline
\end{tabular}




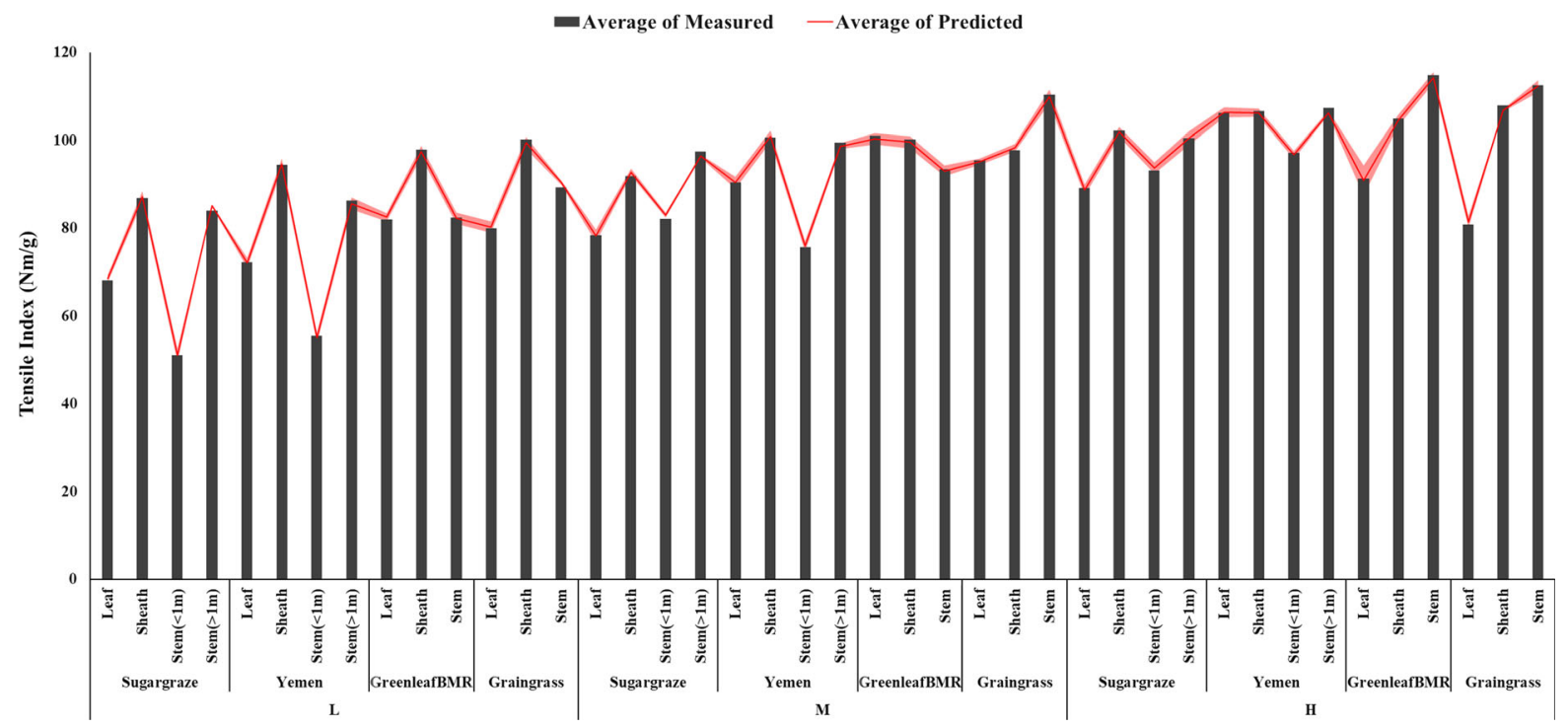

Fig. 3 Measured values of nanopaper tensile index (bars), values predicted by the SVR model (line), and the standard error of the SVR model predictions (line shadow)

Table 6 Effect of mechanical energy level on fibre morphology to nanopaper prediction model accuracy

\begin{tabular}{|c|c|c|c|c|c|c|c|c|}
\hline \multirow{3}{*}{$\begin{array}{l}\text { NRSME } \\
\text { Model }\end{array}$} & \multicolumn{8}{|c|}{ Nanopaper Metrics } \\
\hline & \multicolumn{2}{|c|}{ Young's Modulus } & \multicolumn{2}{|l|}{ Strain } & \multicolumn{2}{|c|}{ Tensile Index } & \multicolumn{2}{|c|}{ Toughness } \\
\hline & MLR & SVR & MLR & SVR & MLR & SVR & MLR & SVR \\
\hline Low & 1.49 & 0.80 & 2.57 & 0.93 & 2.18 & 0.63 & 1.23 & 0.53 \\
\hline Medium & 1.19 & 0.48 & 1.43 & 0.97 & 1.16 & 0.56 & 1.71 & 0.87 \\
\hline High & 0.67 & 0.44 & 2.15 & 0.87 & 1.35 & 0.44 & 2.35 & 0.79 \\
\hline
\end{tabular}

toughness), indicating a higher fibre morphology to nanopaper prediction capability when higher processing energy was applied. For tensile index and Young's modulus, which were the metrics that the SVR model most accurately predicted in the previous section, low energy samples had the highest NRMSE across the energy series, while the high energy samples for these metrics were the most accurately predicted out of all energy levels, nanopaper metrics, and model types.

Validation of model predictions

Following the high level of accuracy achieved for the fibre morphology to nanopaper SVR model, experimental data for an additional HPH validation series was collected to test the fibre morphology to nanopaper model predictions within a new sample population. The HPH validation series extended the processing energy input for CNF production to range from a minimum of one pass at $200 \mathrm{bar}$, to a maximum of 3 passes at 1100 bar. The validation series was performed on an aggregated biomass sample of the Sugargraze variety with all sections combined in equal proportions. The predicted values for each of the four nanopaper metrics, based on the HPH validation series fibre morphology data and the previously established SVR model, was compared to the actual mechanical property results collected from HPH validation series nanopaper samples, to test the degree of overfitting of the initial SVR model. As seen in Table 7, the SVR model is once again more accurate than the MLR model for all nanopaper metrics besides tensile index, with Young's modulus again demonstrated as the most accurate metric for the SVR model. However, the accuracy of the validation series models was significantly lower than the original regression models, with 
Table 7 MLR and SVR model outputs for validation energy series

\begin{tabular}{lllll}
\hline Validation Series Outputs & \multicolumn{2}{l}{ Nanopaper Metrics } & & \\
\cline { 2 - 5 } & Young's Modulus & Strain & Tensile Index & Toughness \\
\hline MLR NRMSE & 1.70 & 2.42 & 1.62 & 2.14 \\
SVR NRMSE & 0.81 & 1.86 & 1.72 & 1.95 \\
\hline
\end{tabular}

NRMSE values ranging from 4.1 to 7.5 times higher across all nanopaper metrics for the validation series.

\section{Discussion}

Parameters influencing nanopaper properties

\section{Influence of kinked fibres}

The relationship between fibre kink and paper properties has rarely been described and has not been extensively elucidated in the literature thus far (Leopold and Thorpe 1968; Guangsheng et al. 2012; Sood and Sharma 2021). For tissue paper applications, the presence of kinked fibres increases material porosity and surface roughness, but negatively impacts the paper density and inter-fibre bond strength (Morais et al. 2021). However, in the case of a cellulose nanofibre system, fibre bundles that have undergone partial microfibrillation through homogenisation may be interpreted as fibre kinks due to limitations in optical resolution, which would be related with an increase in nanopaper strength. Alternatively, fibre kinks have previously been described as deformations induced by mechanical stress rather than by chemical pulping (Aguado et al. 2016). Therefore, their association with nanopaper performance could be related to the increasing energy applied to the fibre bundles over the HPH processing series, which induced fibre deformation.

\section{Influence of fibre width and coarseness}

Unsurprisingly, fibre width and coarseness are inversely correlated with all facets of nanopaper performance, such that less fibrillated materials with higher average fibre width yields lower performing nanopaper. Fibres with a larger width decrease the degree of fibre collapse during paper formation, impacting paper density and strength, in addition to reducing water retention properties (Morais et al. 2021). In addition, the higher size and rigidity of coarse fibres decreases the number of contact points and the bonding strength between fibres. Correspondingly, the wet strength of paper has previously been shown to be inversely proportional to the square of the fibre coarseness (Seth 1995).

\section{Influence of fibre content}

In the context of a cellulose nanofibre suspension, fibre content can be considered a proxy for the degree of fibrillation, such that the higher the number of distinct fibres present within a gram of material infers the disintegration of larger aggregated fibre bundles into smaller fibre structures. Fibre content only relates to the detectable cellulose microfibres (CMF), as a substantial fraction of the total fibre content has been liberated into smaller nanofibres through mechanical processing. Nanofibres that exist below the theoretical detection limit of the MorFi device (Fine element length $<5 \mu \mathrm{m}$ ) are not accounted for in this parameter (Di Giuseppe et al. 2016). To take fibre content as a reliable proxy for the degree of nanofibrillation, an assumption must be made that the shift in micron-scale fibre content with increased processing energy is mirrored in magnitude by the shift in the nanofibre content, which itself is unable to be measured directly with precision and reliability.

Many confounders are present in this parameterfirstly, a significant fraction of the nanofibre population within the sample is hidden from detection (Morais et al. 2021). In addition, with the increase in mechanical processing, the content of micron-scale CMF will firstly increase in number as cellulose bundles are disrupted and partially fibrillated, but subsequently decrease once they are sufficiently fibrillated below the MorFi detection limit, creating a non-linear trend with increasing mechanical energy. In summary, it is impossible to know the true fibre content (percentage of fibres between 100 and $10 \mathrm{~mm}$ 
in length) or fine content of any sample using the MorFi device. However, fibre content still appears to be a promising proxy for the degree of nanofibrillation due to the relatively strong correlation with nanopaper mechanical properties, as demonstrated in Fig. 2a and Table 4.

In a broad sense, this challenge of accurately characterising the true nanofibre content of CNF pulp is pervasive across nanocellulose research (Foster et al. 2018). High resolution microscopy is unreliable due to previously discussed drawbacks such as the analysis of representative samples, the greater length scale of CNF material than the observation window, sufficient image quality, and the time-consuming postprocessing and analysis of images. Fractionation methods such as mechanical screening (Tanaka et al. 2012), gravimetric centrifugation (Ahola et al. 2008), and tube flow fractionation (Haapala et al. 2013) can assess fibre size distribution over multiple length scales, but are limited by their minimum size range for analysis and time-consuming operation. Fractionation and flow cytometry analyses are promising techniques for population level analysis of fibre size distribution, and have the potential to be used as a process quality monitoring and development tool. However, they are excluded from nano-scale particle analysis due to the limited particle size recognition range (Haapala et al. 2013). The MorFi device fits in a similar category to flow cytometry analysis as a potential on-line, high throughput process quality tool for monitoring micron-scale particles within CMFs or CNFs. While optical fibre analysis does not provide true quantitative information on the fibre dimensions and morphology of the material, it may provide valuable insights into the status of the micron scale sub-region of the material at the population level, which can be used to comparatively assess shifts in morphology across different source materials or mechanical processing levels.

\section{Influence of random variation}

Across the different facets of nanopaper performance, fibre morphology parameters didn't correlate as strongly with nanopaper strain at break as they did with other mechanical properties. A hypothesis to explain this is the impact of non-fibre related factors on the strain at break value. A number of microfractures are expected to be imparted to the edges of some nanopaper strips during the sample cutting procedure, which could be a random process or associated with the biochemical composition and rigidity of the nanopaper handsheet itself (Pennells et al. 2021). As such, the number and size of microfractures imparted to the nanopaper strip would disproportionately impact the strain at break result for the tested nanopaper strip.

PCA for population level fibre analysis

PCA has previously been employed to visualise and assess the properties that influence fibre quality (Legland and Beaugrand 2013; García-Gonzalo et al. 2016; Desmaisons et al. 2017). In the case of Desmaisons et al., a PCA methodology was employed to reduce the number of relevant parameters required for subsequent multivariate linear regression (Desmaisons et al. 2017). In the case of Legland and Beaugrand, a PCA methodology was employed to identify highly correlated variables and variable clusters within the fibre morphology data to eliminate redundant variables. This methodology was extended to identify groups of variables that cluster together to generate a hierarchical clustering dendrogram that delineated fibre morphology features based on size, elongation, and tortuosity. This methodology allows for the high resolution morphological characterisation of a diverse fibre population (Legland and Beaugrand 2013). Lastly, in the case of García-Gonzalo et al., a PCA methodology was employed to cluster together different paper properties and the effect of different biomass sources on paper properties (García-Gonzalo et al. 2016).

In this study, PCA was employed to assess and visualise fibre morphology and nanopaper properties from a large population of CNF samples across different biomass types and processing energy levels. Each point represents an individual sample replicate characterised by MorFi fibre morphology or nanopaper mechanical performance. The PCA ellipses represent the region defined by the $68 \%$ normal probability (Prager et al. 2020). The normal probability definition of the ellipse can be adjusted to generate a more or less rigorous ellipse visualisation. Arrows represent the fibre morphology parameters or nanopaper properties determined through CNF pulp and nanopaper characterisation, respectively. The arrow direction represents the correlation between the fibre 

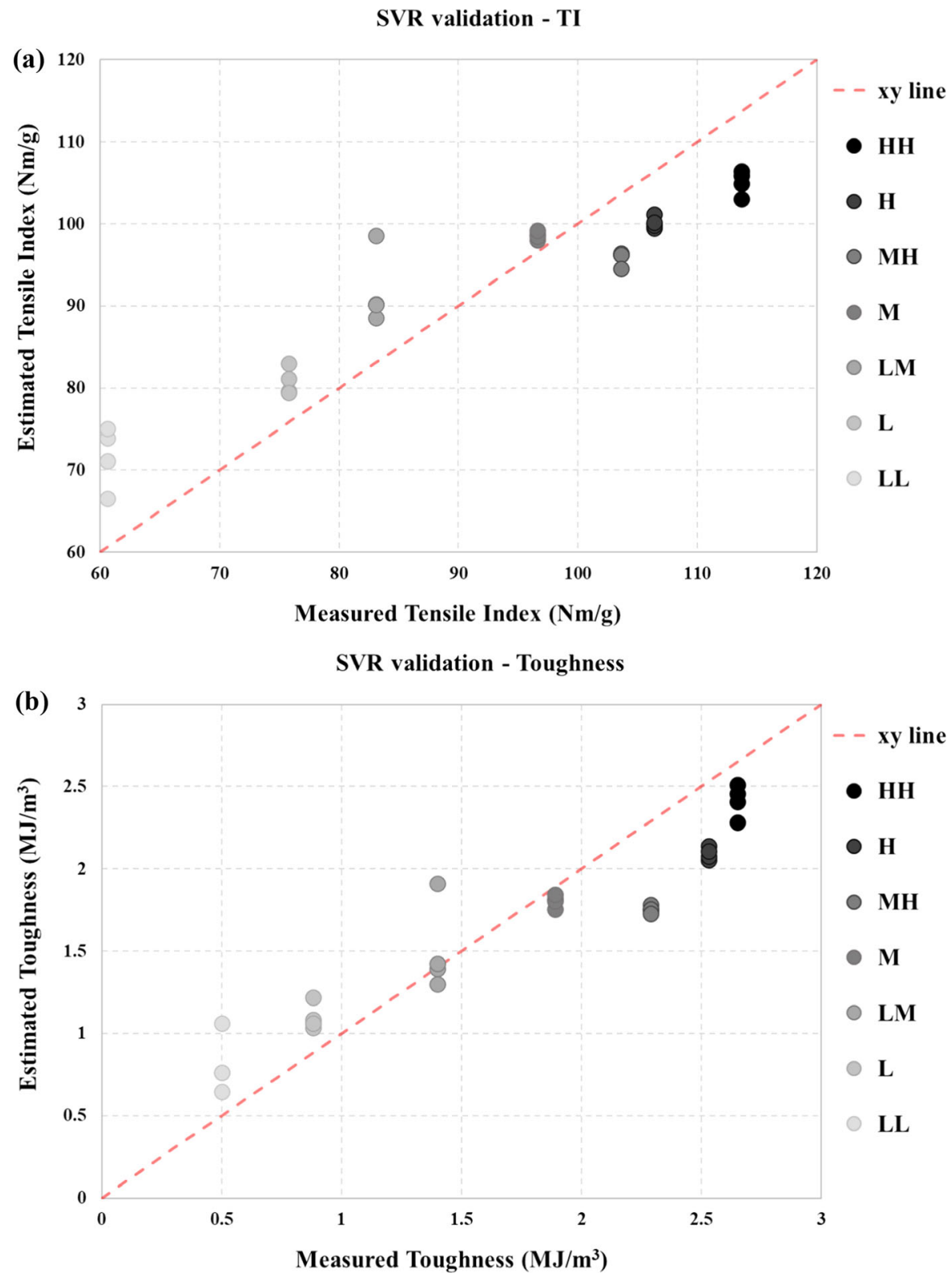

Fig. 4 Nanopaper mechanical properties predicted by the SVR model compared to measured values for: a Tensile index, $\mathbf{b}$ Toughness

morphology parameter/nanopaper properties and the principal component, and the arrow length represents the strength of the relationship between the parameter/ property and the principal component. The strength of this methodology is the visualisation of an array of fibre morphology data at the population level on a single plot, with the elucidation of biomass and processing factors that are associated with different morphology parameters and nanopaper properties through the grouping with probability ellipses.

Predicting nanopaper properties

The overarching goal of this publication is to analyse whether fibre morphology generated from an optical fibre analysis device can be used to predict the quality of cellulose nanofibres in aqueous suspension, without 
having to fabricate and test nanopaper samples. Achieving this goal would provide substantial benefit for industrial processing of nanocellulose, as this would allow for the adoption of an on-line, fast turnaround quality control tool and save time from the fabrication and testing of nanopaper samples. This goal was addressed by analysing fibre morphology and nanopaper mechanical property data using two modelling techniques: Multiple Linear Regression (MLR) and Support Vector Regression (SVR). All fibre morphology parameters generated by the MorFi device were included in the MLR model, as no additional effort is required to gather all data outputs when running this fibre analysis. However, this approach has the potential to lead to model overfitting, which was assessed through model validation. Considering that the NRMSE values were 4.1 to 7.5 times higher for the validation series over the original data series, this indicates that the SVR model was somewhat overfit for the original sample population. The inclusion of all fibre morphology parameters is a potential explanation for the result, with the exclusion of non-significant parameters expected to improve the degree of model overfitting. Subsequent work will investigate the adjusted $\mathrm{R}^{2}$ of the model as an indicator of sufficient parameter inclusion. An additional factor that may reduce model overfitting is further optimisation of SVR hyperparameters.

\section{Effect of energy level on nanopaper predictions}

It is well established that the level of energy applied during mechanical processing of biomass into CNFs influences the fibre morphology and mechanical properties of the resulting materials, as demonstrated by PCA visualisation in Fig. 2. Therefore, it is important to assess the effect of processing energy level on the accuracy of nanopaper predicting models. The results in Table 6 demonstrate that low energy samples had the highest NRMSE values, which indicated that the fibre morphology to nanopaper prediction accuracy was lower for these samples. This result was somewhat unexpected, considering that the MorFi device is attuned to analysing micro-scale CMF that are more likely to be present in higher proportions at low energy conditions. The higher the mechanical energy level, the more likely that microfibres are deconstructed to nano-sized fibres that are outside the detection limit of the device. On the other hand, the nanopaper mechanical properties had a higher distribution between biomass samples at low energy conditions. Homogenisation at the high energy level led to a more homogenised data distribution between biomass samples, which allowed for a more accurate prediction of nanopaper properties for the regression models.

To visualise the accuracy of SVR model for the validation series dataset and further assess the effect of energy level on nanopaper predictions, measured nanopaper values were compared to the SVR estimated values for the data validation series (Fig. 4). Firstly, these results demonstrate that the medium processing energy region is most accurately predicted in the validation series, while the samples at the low and high energy extremes were less accurately predicted. This relationship held for both tensile index and toughness, the latter of which demonstrated a lower prediction accuracy for medium energy samples earlier in Table 6. This suggests that the applicability of the model is lessened when the processing energy conditions are broadened, indicating some degree of overfitting to the specific processing energy conditions of the initial dataset for the SVR model.

\section{Conclusions}

To address the challenges associated with scalable CNF characterisation, this study investigated the relationships between fibre morphology and nanopaper properties for a broad sample population of sorghum biomass. Important fibre morphology parameters elucidated through correlation analysis included the positive correlation between fibre content and fibre kink with nanopaper properties, and the inverse correlation between fibre width and coarseness with nanopaper properties. Regression modelling of the fibre morphology to predict nanopaper properties demonstrated superior predictive power for the machine learning based support vector (SVR) model. The SVR model was further validated through the replication data set over an extended processing energy range, yielding a lower prediction accuracy than the original dataset that implied some degree of model overfitting. This study constitutes a platform for future investigation targeted at predicting nanopaper mechanical properties from the morphological properties of CNF pulp, with a focus on improving model 
generalisability. Ultimately, the development of more accurate and generalisable models for the prediction of nanopaper mechanical properties from morphological data will enable scalable and expedient characterisation of CNF products in the future industrial setting.

Acknowledgments The authors gratefully acknowledge the Australian Government Research Training Program (RTP) scholarship and the University of Queensland's tuition fee offset, along with the Grains Research and Development Corporation (GRDC) Research Scholarship for their support of this research.We appreciate the assistance provided by Yan Luo and Didier Rech from Techpap, France for installation and maintenance of the MorFi device, along with assistance in the interpretation of fibre morphology data.The authors acknowledge the contribution of Dugalunji Aboriginal Corporation on behalf of the Indjalandji-Dhidhanu peoples through use of their equipment and in-kind support.

Funding Open Access funding enabled and organized by CAUL and its Member Institutions.

\section{Declarations}

Conflict of interest This research was supported by the Australian Government Grains Research and Development Corporation (GRDC) Research Scholarship (Ref: UOQ1903005RSX). The funding source had no involvement in the preparation, writing or submission of this article.

Open Access This article is licensed under a Creative Commons Attribution 4.0 International License, which permits use, sharing, adaptation, distribution and reproduction in any medium or format, as long as you give appropriate credit to the original author(s) and the source, provide a link to the Creative Commons licence, and indicate if changes were made. The images or other third party material in this article are included in the article's Creative Commons licence, unless indicated otherwise in a credit line to the material. If material is not included in the article's Creative Commons licence and your intended use is not permitted by statutory regulation or exceeds the permitted use, you will need to obtain permission directly from the copyright holder. To view a copy of this licence, visit http://creativecommons.org/licenses/by/4.0/.

\section{References}

Aguado R, Moral A, López P et al (2016) Morphological analysis of pulps from orange tree trimmings and its relation to mechanical properties. Meas J Int Meas Confed 93:319-326. https://doi.org/10.1016/j.measurement.2016. 06.063

Ahola S, Salmi J, Johansson LS et al (2008) Model films from native cellulose nanofibrils. Preparation, swelling, and surface interactions. Biomacromol 9:1273-1282. https:// doi.org/10.1021/bm701317k
Al-Gharrawi M, Ollier R, Wang J, Bousfield DW (2021) The influence of barrier pigments in waterborne barrier coatings on cellulose nanofiber layers. J Coatings Technol Res. https://doi.org/10.1007/s11998-021-00482-0

Balea A, Blanco A, Delgado-Aguilar M et al (2021) Nanocellulose characterization challenges. BioResources 16:4382-4410. https://doi.org/10.15376/biores.16.2.Balea

Borrell A, Oosterom E Van, George-Jaeggli B, et al (2021) Sorghum. In: Crop physiology case histories for major crops. Academic press, pp 196-221

Campano C, Balea A, Blanco A, Negro C (2020) A reproducible method to characterize the bulk morphology of cellulose nanocrystals and nanofibers by transmission electron microscopy. Cellulose 27:4871-4887. https://doi.org/10. 1007/s10570-020-03138-1

Chinga-Carrasco G (2013) Optical methods for the quantification of the fibrillation degree of bleached MFC materials. Micron 48:42-48. https://doi.org/10.1016/j.micron.2013. 02.005

Desmaisons J, Boutonnet E, Rueff M et al (2017) A new quality index for benchmarking of different cellulose nanofibrils. Carbohydr Polym 174:318-329. https://doi.org/10.1016/j. carbpol.2017.06.032

Di Giuseppe E, Castellani R, Dobosz S et al (2016) Reliability evaluation of automated analysis, 2D scanner, and microtomography methods for measuring fiber dimensions in polymer-lignocellulosic fiber composites. Compos Part A Appl Sci Manuf 90:320-329. https://doi.org/10.1016/j. compositesa.2016.07.020

Dienes D, Kemény S, Egyházi A, Réczey K (2005) Improving the capability of the Schopper-Riegler freeness measurement. Meas J Int Meas Confed 38:194-203. https://doi.org/ 10.1016/j.measurement.2005.07.011

Espinosa E, Rol F, Bras J, Rodríguez A (2020) Use of multifactorial analysis to determine the quality of cellulose nanofibers: effect of nanofibrillation treatment and residual lignin content. Cellulose 27:10689-10705. https://doi.org/ 10.1007/s10570-020-03136-3

Fein K, Bousfield DW, Gramlich WM (2020) Thiol-norbornene reactions to improve natural rubber dispersion in cellulose nanofiber coatings. Carbohydr Polym 250:117001. https:// doi.org/10.1016/j.carbpol.2020.117001

Foster EJ, Moon RJ, Agarwal UP et al (2018) Current characterization methods for cellulose nanomaterials. Chem Soc Rev 47:2609-2679. https://doi.org/10.1039/c6cs00895j

García-Gonzalo E, Santos AJA, Martínez-Torres J et al (2016) Prediction of five softwood paper properties from its density using support vector machine regression techniques. BioResources 11:1892-1904. https://doi.org/10.15376/ biores.11.1.1892-1904

Guangsheng Z, Chao X, Yuejun L, Jinping Q (2012) Rheological behavior and cell morphology of foamed waste paper pulp/ high density polyethylene composites. Appl Mech Mater 200:316-320. https://doi.org/10.4028/www. scientific.net/AMM.200.316

Guay D, Sutherland NR, Rantanen W et al (2005) Comparison of fiber length analyzers. TAPPI Pract Papermak Conf 2005:413-442

Haapala A, Laitinen O, Karinkanta P et al (2013) Optical characterisation of size, shape and fibrillarity from 
microfibrillar and microcrystalline cellulose, and fine ground wood powder fractions. Appita J 66:331-339

Hervy M, Santmarti A, Lahtinen P et al (2017) Sample geometry dependency on the measured tensile properties of cellulose nanopapers. Mater Des 121:421-429. https://doi.org/10. 1016/j.matdes.2017.02.081

Hirn U, Bauer W (2006) A review of image analysis based methods to evaluate fiber properties. Lenxinger Berichte 86:96-105

International Organization for Standardization (2001) BS EN ISO 5269-2:2001-Pulps. Preparation of laboratory sheets for physical testing. Rapid-Kothen method.

Kangas H, Lahtinen P, Sneck A et al (2014) Characterization of fibrillated celluloses. A short review and evaluation of characteristics with a combination of methods. Nord Pulp Pap Res J 29:129-143. https://doi.org/10.3183/npprj-201429-01-p129-143

Koppolu R, Lahti J, Abitbol T et al (2019) Continuous processing of nanocellulose and polylactic acid into multilayer barrier coatings. ACS Appl Mater Interfaces 11:11920-11927. https://doi.org/10.1021/acsami.9b00922

Krishnamachari P, Hashaikeh R, Tiner M (2011) Modified cellulose morphologies and its composites; SEM and TEM analysis. Micron 42:751-761. https://doi.org/10.1016/j. micron.2011.05.001

Kumar V, Bollström R, Yang A et al (2014) Comparison of nano- and microfibrillated cellulose films. Cellulose 21:3443-3456. https://doi.org/10.1007/s10570-014-03575

Kumar V, Elfving A, Koivula H et al (2016a) Roll-to-roll processed cellulose nanofiber coatings. Ind Eng Chem Res 55:3603-3613. https://doi.org/10.1021/acs.iecr.6b00417

Kumar V, Nazari B, Bousfield D, Toivakka M (2016b) Rheology of microfibrillated cellulose suspensions in pressuredriven flow. Appl Rheol 26:24-34. https://doi.org/10.3933/ applrheol-26-43534

Kumar V, Koppolu VR, Bousfield D, Toivakka M (2017) Substrate role in coating of microfibrillated cellulose suspensions. Cellulose 24:1247-1260. https://doi.org/10. 1007/s10570-017-1201-5

Kumar V (2018) Roll-to-Roll Processing of Nanocellulose into Coatings. Åbo Akademi

Lacerda TM, Zambon MD, Frollini E (2013) Effect of acid concentration and pulp properties on hydrolysis reactions of mercerized sisal. Carbohydr Polym 93:347-356. https:// doi.org/10.1016/j.carbpol.2012.10.039

Larsson PT, Lindström T, Carlsson LA, Fellers C (2018) Fiber length and bonding effects on tensile strength and toughness of kraft paper. J Mater Sci 53:3006-3015. https://doi. org/10.1007/s10853-017-1683-4

Legland D, Beaugrand J (2013) Automated clustering of lignocellulosic fibres based on morphometric features and using clustering of variables. Ind Crops Prod 45:253-261. https://doi.org/10.1016/j.indcrop.2012.12.021

Leopold B, Thorpe J (1968) Effect of pulping on strength properties of dry and wet pulp fibres from Norway spruce. Tappi J 51:

Li MC, Wu Q, Song K et al (2015) Cellulose nanoparticles: structure-morphology-rheology relationships. ACS Sustain Chem Eng 3:821-832. https://doi.org/10.1021/ acssuschemeng. 5 b00144
Li T, Chen C, Brozena AH et al (2021) Developing fibrillated cellulose as a sustainable technological material. Nature 590:47-56. https://doi.org/10.1038/s41586-020-03167-7

Lin B, He B, Liu Y, Ma L (2014) Correlation analysis for fiber characteristics and strength properties of softwood kraft pulps from different stages of a bleaching fiber line. BioResources 9:5024-5033. https://doi.org/10.15376/ biores.9.3.5024-5033

Madani A, Kiiskinen H, Olson JA, Martinez DM (2011) Paper physics: fractionation of microfibrillated cellulose and its effects on tensile index and elongation of paper. Nord Pulp Pap Res J 26:306-311. https://doi.org/10.3183/npprj-201126-03-p306-311

Moon RJ, Martini A, Nairn J et al (2011) Cellulose nanomaterials review: structure, properties and nanocomposites. Chem Soc Rev 40:3941-3994. https://doi.org/10.1039/ $\mathrm{c0cs00108b}$

Morais P, Carta AMMS, Amaral ME, Curto JMR (2021) Micro/nano-fibrillated cellulose (MFC/NFC) fibers as an additive to maximize eucalyptus fibers on tissue paper production. 28: 6587-6605. https://doi.org/10.1007/ s10570-021-03912-9

Moral A, Monte MC, Cabeza E, Blanco A (2010) Morphological characterization of pulps to control paper properties. Cellul Chem Technol 44:473-480

Moser C, Lindström ME, Henriksson G (2015) Toward industrially feasible methods for following the process of manufacturing cellulose nanofibers. BioResources 10:2360-2375. https://doi.org/10.15376/biores.10.2.23602375

Motamedian HR, Halilovic AE, Kulachenko A (2019) Mechanisms of strength and stiffness improvement of paper after PFI refining with a focus on the effect of fines. Cellulose 26:4099-4124. https://doi.org/10.1007/s10570-01902349-5

Nasser RA, Hiziroglu S, Abdel-Aal MA et al (2015) Measurement of some properties of pulp and paper made from date palm midribs and wheat straw by soda-AQ pulping process. Meas J Int Meas Confed 62:179-186. https://doi.org/ 10.1016/j.measurement.2014.10.051

Nazari-Nasrabad B (2015) New applications for cellulose nanofibers: Rheological challenges. The university of maine

Oluwafemi OA, Sotannde OA (2007) The relationship between fibre characteristics and pulp-sheet properties of Leucaena leucocephala ( Lam.) de wit. Middle-East J Sci Res 2:63-68

Pääkko M, Ankerfors M, Kosonen H et al (2007) Enzymatic hydrolysis combined with mechanical shearing and highpressure homogenization for nanoscale cellulose fibrils and strong gels. Biomacromol 8:1934-1941. https://doi.org/10. 1021/bm061215p

Pande H, Roy DN (1998) Influence of fibre morphology and chemical composition on the papermaking potential of kenaf fibres: a look at what attributes affect tensile strength. Pulp Pap Canada 99:31-34

Pedregosa F, Weiss R, Brucher M (2011) Scikit-learn: machine learning in Python. J Mach Learn Res 12:2825-2830

Peng Y, Gardner DJ, Han Y (2012) Drying cellulose nanofibrils: in search of a suitable method. Cellulose 19:91-102. https://doi.org/10.1007/s10570-011-9630-z 
Pennells J, Cruickshank A, Godwin ID, Martin DJ (2021) Sorghum as a novel biomass for the sustainable production of cellulose nanofibers. Ind Crop Prod. https://doi.org/10. 1016/j.indcrop.2021.113917

Prager CM, Boelman NT, Eitel JUH et al (2020) A mechanism of expansion: arctic deciduous shrubs capitalize on warming-induced nutrient availability. Oecologia 192:671-685. https://doi.org/10.1007/s00442-019-045868

Qing Y, Sabo R, Zhu JY et al (2013) A comparative study of cellulose nanofibrils disintegrated via multiple processing approaches. Carbohydr Polym 97:226-234. https://doi.org/ 10.1016/j.carbpol.2013.04.086

R Core Team (2021) R: a language and environment for statistical computing. R Found. Stat. Comput.

Ritter A, Muñoz-Carpena R (2013) Performance evaluation of hydrological models: statistical significance for reducing subjectivity in goodness-of-fit assessments. J Hydrol 480:33-45. https://doi.org/10.1016/j.jhydrol.2012.12.004

Rol F, Banvillet G, Meyer V et al (2018) Combination of twinscrew extruder and homogenizer to produce high-quality nanofibrillated cellulose with low energy consumption. J Mater Sci 53:12604-12615. https://doi.org/10.1007/ s10853-018-2414-1

Rol F, Belgacem N, Meyer V et al (2019) Production of fireretardant phosphorylated cellulose fibrils by twin-screw extrusion with low energy consumption. Cellulose 26:5635-5651. https://doi.org/10.1007/s10570-01902447-4

Saito T, Kimura S, Nishiyama Y, Isogai A (2007) Cellulose nanofibers prepared by TEMPO-mediated oxidation of native cellulose. Biomacromol 8:2485-2491. https://doi. org/10.1021/bm0703970

Sanchez-Salvador JL, Monte MC, Batchelor W et al (2020) Characterizing highly fibrillated nanocellulose by modifying the gel point methodology. Carbohydr Polym 227:115340. https://doi.org/10.1016/j.carbpol.2019. 115340

Seth RS (1995) Effect of fiber length and coarseness on the tensile strength of wet webs: a statistical geometry explanation. Tappi J 78:99-102

Shimizu M, Saito T, Nishiyama Y et al (2016) Fast and robust nanocellulose width estimation using turbidimetry.
Macromol Rapid Commun 37:1581-1586. https://doi.org/ 10.1002/marc.201600357

Silva LE, dos Santos A de A, Torres L, et al (2021) Redispersion and structural change evaluation of dried microfibrillated cellulose. Carbohydr Polym 252:. doi: https://doi.org/10. 1016/j.carbpol.2020.117165

Sood S, Sharma C (2021) Study on fiber furnishes and fiber morphological properties of commonly used indian food packaging papers and paperboards. Cellul Chem Technol 55:125-131

Tanaka A, Seppänen V, Houni J et al (2012) Nanocellulose characterization with mechanical fractionation. Nord Pulp Pap Res J 27:689-694. https://doi.org/10.3183/NPPRJ2012-27-04-p689-694

Tang C, Chen Y, Luo J et al (2019) Pickering emulsions stabilized by hydrophobically modified nanocellulose containing various structural characteristics. Cellulose 26:7753-7767. https://doi.org/10.1007/s10570-01902648-x

TAPPI Standard (2006) T 494 om-01, Tensile properties of paper and paperboard (using constant rate of elongation apparatus)

Tayeb AH, Tajvidi M, Bousfield D (2020) Paper-based oil barrier packaging using lignin-containing cellulose nanofibrils. Molecules. https://doi.org/10.3390/ molecules 25061344

Turunen M, Le Ny C, Tienvier T, Niinimaki J (2005) Comparison of fibre morphology analysers. Appita Technol Innov Manuf Environ 58:28-32. https://doi.org/10.3316/ informit.633632227819514

Varanasi S, He R, Batchelor W (2013) Estimation of cellulose nanofibre aspect ratio from measurements of fibre suspension gel point. Cellulose 20:1885-1896. https://doi.org/ 10.1007/s10570-013-9972-9

Yuan T, Zeng J, Wang B et al (2021) Cellulosic fiber: mechanical fibrillation-morphology-rheology relationships. Cellulose 28:7651-7662. https://doi.org/10.1007/ s10570-021-04034-y

Publisher's Note Springer Nature remains neutral with regard to jurisdictional claims in published maps and institutional affiliations. 